\title{
CONTRACTIONS OF CONVEX SETS
}

\author{
ROBERT E. JAMISON
}

\begin{abstract}
In this paper it is shown that, in a vector space over any ordered field, a noninfinitesimal contraction of a convex set $K$ can be written as an intersection of translates of $K$.
\end{abstract}

A subset $K$ of a vector space over a totally ordered field is called convex provided $\lambda x+(1-\lambda) y$ is in $K$ whenever $x$ and $y$ are in $K$ and $\lambda$ is a scalar such that $0 \leqslant \lambda \leqslant 1$. Recalling that any ordered field $F$ has characteristic zero, and hence contains a copy of the rational numbers, we shall say that a positive element $\mu$ in $F$ is infinitesimal if $\mu<r$ for all positive rational numbers $r$. (For further discussion and examples of ordered fields, see [1, Chapter 13].) In this note we shall prove the following intersection theorem:

THEOREM. Suppose $K$ is a convex set in a vector space over an ordered field and $\mu$ is a positive scalar less than 1. If $\mu$ is not infinitesimal, then, for some set $T$ of vectors,

$$
\mu K=\bigcap\{K+t: t \in T\} .
$$

It is easy to see that this result is plausible by considering either a square or a triangle in the plane, or in fact, any closed convex set. Difficulties arise, however, in the case of a convex set which includes only a portion of its boundary-say, the open unit disk together with the points of its circumference with rational $x$-coordinate.

ProOF OF THE THEOREM. Let $\mu$ be a noninfinitesimal positive scalar less than 1. Set $P=\mu K$ and suppose $q$ is a point not in $P$. To prove the theorem, we must find a vector $t$ such that $P \subseteq K+t$ but $q \notin K+t$. We distinguish two cases:

Case I. For all $x$ in $P$, we have $q+\mu(x-q) \in P$. Let $t=\left(1-\mu^{-1}\right) q$ so that

$$
K+t=\mu^{-1} P+\left(1-\mu^{-1}\right) q=q+\mu^{-1}(P-q) .
$$

The vectors in $P-q$ are all nonzero since $q$ is not in $P$. Thus $q$ is not in $K+t$. But if $x$ is in $P$, then $q+\mu(x-q) \in P$ by the case hypothesis, so that $x \in q+\mu^{-1}(P-q)=K+t$. Thus $P \subseteq K+t$.

\footnotetext{
Received by the editors August 5, 1975 and, in revised form, March 24, 1976.

AMS (MOS) subject classifications (1970). Primary 52A05; Secondary 15A03, 12 J15.

Key words and phrases. Contraction, convex set, intersection theorem, ordered field, translation, vector space.
} 
Case II. There is a point $p$ in $P$ such that $q+\mu(p-q) \notin P$. Note that if $0<\lambda<\mu$, then the segment from $q+\lambda(p-q)$ to $q+(p-q)$ contains $q+\mu(p-q)$. Since $q+(p-q)=p \in P$ and $P$ is convex, the assumption on $p$ forces $q+\lambda(p-q) \notin P$.

Now since $\mu$ is not infinitesimal, the positive integral powers of $1-\mu$ become ultimately smaller than any preassigned positive rational number and, hence, smaller than any preassigned positive noninfinitesimal. Hence, $(1-\mu)^{n}$ $<\mu$ for some sufficiently large positive integer $n$. By the preceding note, $q+(1-\mu)^{n}(p-q)$ cannot belong to $P$.

Let $m$ be the smallest positive integer such that $q+(1-\mu)^{m}(p-q) \notin P$, and set $v=q+(1-\mu)^{m-1}(p-q)$. (Here $v=p$ if $m=1$.) Then $v$ belongs to $P$. If $x$ is any point in $P$, then $(1-\mu) \nu+\mu x \in P$ since $P$ is convex. Whence

$$
x \in \mu^{-1}(P-(1-\mu) v)=K+\left(1-\mu^{-1}\right) v .
$$

Thus letting $t=\left(1-\mu^{-1}\right) v$, we have $P \subseteq K+t$. But

$$
\mu q+(1-\mu) v=q+(1-\mu)(v-q)=q+(1-\mu)^{m}(p-q) \notin P .
$$

Consequently, $q$ does not belong to

$$
\mu^{-1}(P-(1-\mu) v)=K+\left(1-\mu^{-1}\right) v=K+t .
$$

This completes the proof of the theorem.

We conclude with a simple one-dimensional example to show that the result cannot be extended to include infinitesimal contractions. Let $F$ be an ordered field which contains a positive infinitesimal element $\delta[1$, p. 70]. Take the convex set $K=\{x \in F: x \geqslant 0$ and for some integer $n, x \leqslant n\}$. If $\lambda$ $\in F$ such that $\delta K \subseteq K-\lambda$, then $0 \in K-\lambda$ since $0 \in \delta K$. Thus $\lambda \in K$, so $\lambda+1$ must also be in $K$. Whence $1 \in K-\lambda$. Thus 1 belongs to every translate of $K$ containing $\delta K$, but 1 does not belong to $\delta K$ since $\delta$ is infinitesimal.

ACKNOWLEDGEMENT. The author would like to acknowledge the referee's suggestions of refinements in the proof of the theorem.

\section{BIBLIOGRAPHY}

1. L. Gillman and M. Jerison, Rings of continuous functions, Van Nostrand, Princeton, N. J., 1960. MR 22 \#6994. 29631

Department of Mathematical Sciences, Clemson University, Clemson, Sourth Carolina

Current address: Mathematisches Institut, Universität Erlangen, 852 Erlangen, Federal Republic of Germany 